\title{
Social norms in adolescent risk engagement and recommendation
}

\author{
Simon Ciranka ${ }^{1,2 *}$ (iD) and Wouter van den Bos ${ }^{1,2,3}$ \\ 'Max Planck institute for Human Development, Berlin, Germany \\ ${ }^{2}$ Max Planck UCL Centre for Computational Psychiatry and Ageing Research, Berlin, \\ Germany \\ ${ }^{3}$ University of Amsterdam, Netherlands
}

\begin{abstract}
Social influence is an important determinant of adolescent risk-taking, but little is known about how social norms contribute to adolescents' tendency to recommend and engage in risky behaviours. Using the Adolescent Risk-taking Questionnaire, we assessed subjects' $(n=198$, age $=10-26)$ propensity to engage in and recommend risk-taking as well as their perception of risk norms. Adolescents recommended risktaking more often compared to children and young adults. Perceived social norms were the most important factor predicting engagement in risky behaviours, and adolescents perceived risk-taking to be more normative than children or adults. Our findings highlight two mechanisms that contribute to adolescent risk-taking, active recommendation and perceived social norms. On this basis, we discuss potential means to attenuate excessive adolescent risk-taking.
\end{abstract}

\section{Statement of Contribution}

What is already known on this subject?

- Adolescents are very sensitive towards social information.

- Adolescent risk-taking is tightly connected to social contexts.

- Little is known about how adolescents perceive and endorse social risk norms.

\section{What does this study add?}

- Adolescents are sensitive to social norms when considering risk engagement, but not more than other age groups.

- Adolescents overestimate frequency of others' risky behaviour.

- Adolescents are more likely to recommend risky behaviours compared to other age groups.

\footnotetext{
This is an open access article under the terms of the Creative Commons Attribution-NonCommercial License, which permits use, distribution and reproduction in any medium, provided the original work is properly cited and is not used for commercial purposes.

*Correspondence should be addressed to Simon Ciranka, Lentzeallee 94, 14195 Berlin, Germany (email: Ciranka@mpibberlin.mpg.de).
} 


\section{Background}

The most important determinants of adolescent risky and deviant behaviour are thought to be social. For instance, the number of teenagers in a car is positively correlated with the likelihood of an accident (Carter, Bingham, Zakrajsek, Shope, \& Sayer, 2014), teenage crime is more often committed in groups than alone (Shulman, Steinberg, \& Piquero, 2013), and peers play a pivotal role in adolescents' decisions to start smoking or use illicit drugs (Ragan, 2016). Despite the strong correlations between social factors and adolescent risk-taking, there still is much to learn about why adolescents seemingly take more risks when they find themselves in social contexts. While it was suggested that adolescents may simply join peer groups with similar attitudes (Kandel, 1978; Young, Rebellion, Barnes, \& Weerman, 2014), experimental evidence suggests that adolescents actively influence each other's risky behaviour and risk attitudes (Albert \& Steinberg, 2011; Blakemore \& Robbins, 2012; Brechwald \& Prinstein, 2011; Chein, Albert, O'Brien, Uckert, \& Steinberg, 2011; Helfinstein, Mumford, \& Poldrack, 2015; Knoll, MagisWeinberg, Speekenbrink, \& Blakemore, 2015; Molleman, Kanngiesser, \& van den Bos, 2019; Schriber \& Guyer, 2016; Shulman et al., 2016; Somerville, 2013; van den Bos, Crone, Meuwese, \& Güroğlu, 2018; van Duijvenvoorde, Peters, Braams, \& Crone, 2016).

Most theories explain heightened adolescent risk-taking in social contexts by assuming that adolescents process social information differently than adults or children (Blakemore, 2008; Steinberg, 2008). Some state that merely being in a social situation itself leads adolescents to focus more on the rewards associated with risky behaviour and thus leads to increased risk-taking (Shulman et al., 2016). Other standpoints emphasize that adolescents are particularly sensitive towards social information in general (Blakemore, 2008). Experimental research based on these theories usually involves disclosing others' decisions to subjects, or manipulating peer presence, and examining how social context influences behaviour (Blankenstein, Crone, van den Bos, \& van Duijvenvoorde, 2016; Braams, Davidow, \& Somerville, 2019; Chein et al., 2011; Ciranka \& van den Bos, 2019; Knoll et al., 2015; Molleman et al., 2019; Reiter, Suzuki, O'Doherty, Li, \& Eppinger, 2019; Somerville et al., 2013, 2018).

It is easy to imagine how 'black sheep' pressure their friends to take risks or break the law, but social influence in the real world likely results from at least three processes - (1) copying observed behaviours (Bandura, Ross, \& Ross, 1961), (2) active peer pressure (Shepherd, Lane, Tapscott, \& Gentile, 2011), (3) conforming to perceived social norms (Cialdini \& Goldstein, 2004) - or a combination of the three.

Although evidence suggests that adolescents are more likely than adults but less likely than children to directly copy observed peer behaviour (Blankenstein et al., 2016; Braams et al., 2019; Ciranka \& van den Bos, 2019), little is known about adolescents' tendency to actively recommend risky behaviours. While it is long-established that peer pressure has a strong impact on adolescent deviant behaviour (Brown, Clasen, \& Eicher, 1986), whether adolescents are more likely to actively recommend risk-taking has received less attention. However, the numerous reports of increased risk-taking in peer contexts during adolescence and the close connection between risk-taking and peer encouragement (Brechwald \& Prinstein, 2011; Brown et al., 1986; Shepherd et al., 2011), lead us to hypothesize that adolescents would be more likely than children or young adults to recommend risky behaviours.

It is also not known to what extent social norms contribute to engagement in risky behaviour across adolescence. Although studies have shown that adolescents, like adults, conform to social norms when taking risks (Ragan, 2016; van de Bongardt, Reitz, Sandfort, 
\& Deković, 2015), it remains unclear if sensitivity to social norms peaks in adolescence in direct comparison to other age groups. Theory has it that peer approval and belonging are especially motivating for adolescents (Blakemore \& Mills, 2014; Crone \& Dahl, 2012; Telzer, 2016), and conforming to peer norms is a straightforward way to gain approval and increase belonging (Blakemore, 2008; Blakemore \& Mills, 2014; Cialdini \& Goldstein, 2004; Ciranka \& van den Bos, 2019; Crone \& Dahl, 2012; Pickett, Gardner, \& Knowles, 2004). For this reason, it is to expect that, relative to children and young adults, adolescent risk-taking would be more strongly influenced by social norms (Albert \& Steinberg, 2011; Blakemore \& Mills, 2014; Nelson, Jarcho, \& Guyer, 2016).

In sum, adolescent risk-taking occurs more often than not in social contexts. Despite the big role of social information in adolescence, not much is known about whether adolescents are also more likely to actively recommend risk-taking than are people of other ages. It is also not known to what extent they are more sensitive to perceived risk norms (in absence of overt peer behaviour). Addressing these questions will help to understand why and when adolescents are more likely to engage in risky behaviours than other age groups and how the interaction between social factors and individual judgements contribute to the potential of adolescent risk-taking to escalate. In this study, we therefore investigate two main questions: (1) are adolescents more likely than adults and children to actively encourage risky behaviours? (2) does the relationship between perceived social norms and the engagement in risky behaviours peak in adolescence? To answer these questions, we assessed our participants' propensity to engage in and recommend risky behaviours, as well as their perceptions of how frequently others engage in those behaviours as a measure of their perception of descriptive social norms.

\section{Methods}

We invited $N=198$ (90 female) German subjects aged from 10 to 26 years $(M=16.15$, $S D=4.6$ ) to our laboratory for completing multiple social experiments (L. Molleman, S. Ciranka, \& W. van den Bos, in prep), an assessment of their general cognitive (CFT-20; Weiß, 1998), and emotional intelligence (Reading the mind in the eyes; Megías-Robles et al., 2020) as well as a survey study. We detail the results of the survey study in this paper. Subjects were recruited from our institute's local database from which, after a telephone screening, all healthy minors and 64 legal adults were invited. While our statistical analysis treats age as a continuous variable, in the text we refer to subjects between 12 and 21 years as adolescents, thus adopting a similar heuristic as Shulman et al., (2016). Other than Shulman et al., (2016), we label 10- to 12-year-olds as children because puberty (and therefore the onset of adolescence) related physiological changes are greatest on average at 12.8 years of age (Petersen, Crockett, Richards, \& Boxer, 1988). The paper and discussion focus on adolescence, but we also recruited children and young adults, otherwise we would not be able to make statements about adolescents in comparison with other age groups.

We asked subjects to complete the German version of the Adolescent Risk-Taking Questionnaire (Gullone, Moore, Moss, \& Boyd, 2000). The ARQ consists of 22 examples for risky behaviours (see Tables S1 and S2). Respondents were asked to indicate on a scale ranging from 0 to 4 how risky they perceive each behaviour to be and how often they engage in each behaviour described. Additionally, we asked participants to evaluate the questionnaire scenarios on two dimensions not included in the original inventory: how many of their peers engaged in this behaviour (from $0=$ very few to $4=$ very many) and 
how recommendable they considered behaviour to be to someone in general (from $0=$ not at all recommendable to $4=$ highly recommendable). Each of the four surveys was interleaved with other experiments and presented in random order. The authors of the ARQ identified four factors of the questionnaire: thrill-seeking behaviours, reckless behaviours, rebellious behaviours, and antisocial behaviours. These factors can be further collapsed to describe desirable (thrill-seeking) and undesirable (reckless, rebellious, and antisocial) behaviours. An adult study similar in the design to this study found opposite patterns of engagement and recommendation for 'desirable' and the 'undesirable' risk behaviours (Helfinstein et al., 2015). Further, it has recently been proposed that this distinction provides a more relevant framing for understanding adolescent risk-taking (Duell \& Steinberg, 2019), we therefore applied this distinction in our analyses as well. Participants completed the survey individually on computers using jsPsych (de Leeuw, 2015). All subjects - and, if they were underage, their parents - gave their informed consent to participate in this study, in accordance with the Declaration of Helsinki. Prior to any data collection, the present study was approved by our internal ethics committee (protocol number: A 2018/23).

\section{Statistical analysis}

Responses were analysed using Bayesian multivariate generalized mixed models with random intercepts for subjects and questionnaire subscales, utilizing a cumulative link and fitted via the No-U-Turn sampler (NUTS) implemented in stan (Carpenter et al., 2017), using the brms package (Bürkner, 2017) for R. Dependent variables were the reported propensities to engage in a behaviour and to recommend the same behaviour. The multivariate model assumed that the dependent variables were correlated within subjects. Predictor variables were participant age, ratings of the riskiness of the behaviour in question, and perceived social norms, as quantified by an estimate of how many friends and acquaintances engaged in the behaviour. Developmental changes in social influence were modelled as the interaction between age and perceived social norms. We adjusted for gender differences. We ran two separate regressions: for desirable and undesirable risks. For each regression, we ran six chains with 20,000 samples each and set a warmup of 10,000 samples in order to ensure an effective sample size of at least 10,000 samples for each predictor. We performed approximate leave-one-out cross-validation on the loglikelihood from the model posteriors (Vehtari, Gelman, \& Gabry, 2017) to and thus compare alternative explanations to the one we mainly consider in this article with regard to their predictive accuracy.

Specifically, we asked whether the age predictors should be included and if so, if they should be linear, quadratic, or both. Further, we post-hoc explored the possibility that general or social intelligence constitutes a confound in our results by adding the outcomes of age appropriate versions of the 'CFT-20' and of the 'reading the mind in the eyes' test as main effects and their interactions with age in another regression model.

The quadratic age regressor as constructed by us had its maximum at 18 years of age. Thus, a higher estimate of this regressors weight suggests stronger propensities during late adolescence. This specific peak was the result from creating orthogonal predictors for linear and quadratic age using R's 'poly' function and the age range of our sample. The predictor is sensitive to a recently reported peak in risk-taking propensities during late adolescence (Steinberg et al., 2018). In a post-hoc analysis, we explored age effects in the perception of social norms by using subjects' norm-estimates as a dependent variable, with a quadratic age predictor, random intercepts for subjects and subscales, and a cumulative link. 
We report the mean of the posterior distribution and the two-sided 95\% credible intervals (CI) around each mean of the regression weights. Importantly, the Bayesian CIs which we report allow their direct comparison and interpretation as mean of and uncertainty about the regression weights; an interpretation that is unwarranted when using frequentist methods (Morey, Hoekstra, Rouder, Lee, \& Wagenmakers, 2016; Rouder, Morey, Verhagen, Province, \& Wagenmakers, 2016). The inference criteria about the magnitude of and difference in magnitude between predictors are as follows: If the credible interval includes zero, the contribution of a predictor to the outcome is considered negligible. If their credible intervals overlap, the difference between predictors is considered negligible.

To determine whether the difference in engagement and recommendation was substantial, we tested the hypothesis that both measures differed with respect to a normally distributed effect size, $\delta$. To establish the magnitude of this effect size, we fitted a Bayesian model, assuming

$$
\Delta_{\text {eng-rec }} \sim \mathscr{N}\left(\delta_{i} * \sigma_{i}, \sigma_{i}^{2}\right),
$$

where $\Delta_{\text {eng-rec }}$ denotes the difference between propensity to engage and propensity to recommend, $\sigma$ is the standard deviation, and $i$ is an index for questionnaire subscale. We defined a region of practical equivalence, or ROPE (Kruschke, 2018), for $\delta$ with boundaries $[-0.1,0.1]$, which corresponds to the generally adopted definition of a small effect size. We treated effect sizes as practically equivalent to a null effect (indicating that there is no difference in recommendation and engagement) when the two-sided $95 \%$ CI of $\delta$ overlapped with the ROPE. By defining a ROPE over the range of a small effect size, we explicitly adopt a conservative inference strategy in order to protect us from overestimating the credibility of this difference. Finally, we compute Cronbachs' alpha of all scales analysed in order to judge the reliability of the survey instrument that we chose for the study.

\section{Power}

We use two methods to judge whether the effects reported by us are sufficiently large to be reliably detected with the analysis strategy we chose. First, we compute Bayes factors for all credible regression coefficients by comparing the posterior estimates to the priors implemented in brms (for a discussion on Bayes factors and frequentist power, see Wagenmakers, Lodewyckx, Kuriyal, \& Grasman, 2010). Bayes factors quantify the strength of evidence in favour of the reported effects, given the uncertainty about the effect size. Second, we simulate 1,000 new datasets based on the full posterior of the estimated regression weights. We then proceed to fit the same regression model again to each of these synthetic datasets, using four chains and 2,000 samples per chain and then evaluate the probability with which we can credibly replicate the effect based on the effect size, uncertainty about that effect size and our sample size (post-hoc power).

\section{Results}

We investigated the developmental and social correlates of participants' propensity to recommend and engage in risky behaviours. Overall, subjects were more inclined to engage in risky behaviours than they were to recommend them to others, when the 


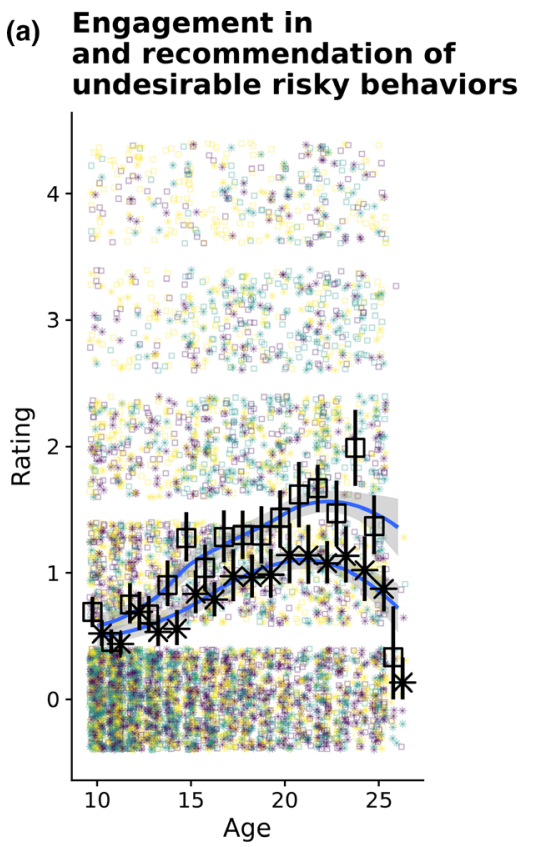

(c) Engage > recommend undesirable

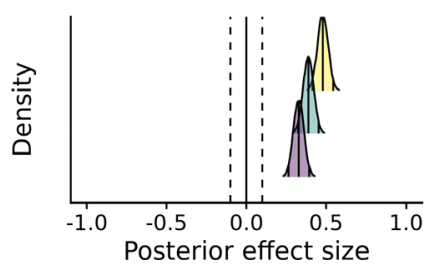

(b) Engagement in
and recommendation of
desirable risky behavior

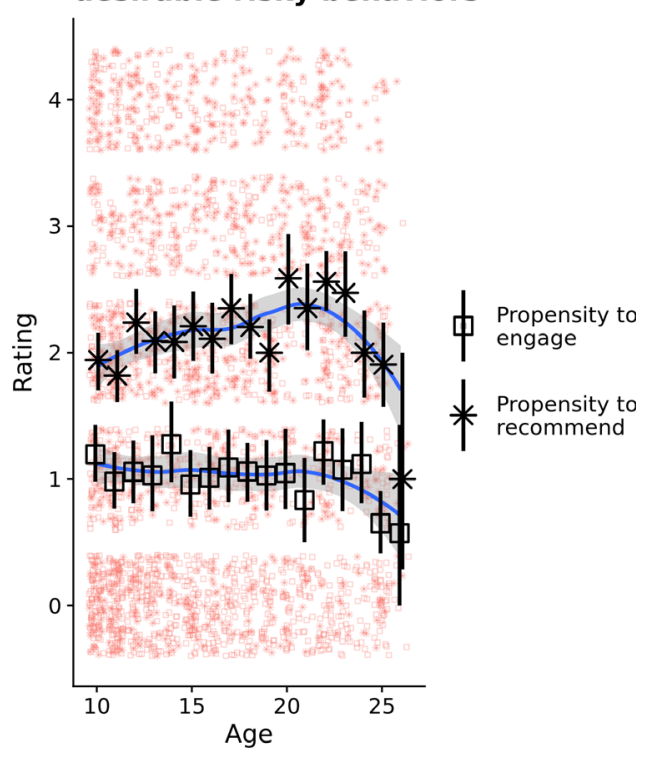

(d) Engage > recommend desirable

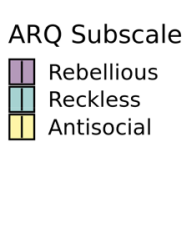

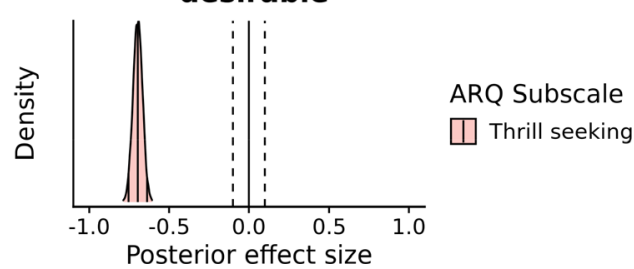

Figure I. Propensity to engage in and recommend risk behaviours by age. The upper panel shows the difference between participants' propensity to engage in and to recommend the (a) undesirable and (b) desirable behaviours. The lower panel shows the posterior effect size distribution of this difference by ARQ subscale. Vertical lines denote the region of practical equivalence of the effect size, with boundaries $[-0.1,0.1]$, for (c) undesirable and (d) desirable risky behaviours. It can be seen that while undesirable subscales (c) are not credibly different from one another, they jointly show the opposite pattern of the desirable subscale (d). [Colour figure can be viewed at wileyonlinelibrary.com]

scenarios described undesirable risk-taking. When the scenarios described desirable risktaking, the opposite pattern was observed (Figure 1).

We note that the inventory we used was sufficiently reliable as indicated by Cronbachs' alpha of .76 for both, engagement and recommendation (item level characteristics in the supplement).

In what follows, we summarize the results of the regression analysis.

To begin with, we used approximate leave-one-out cross-validation to determine the predictive accuracy of the regression models containing different age predictors and potential confounds (Vehtari et al., 2017). This procedure yielded that the regression models containing quadratic and linear age as predictors but no measures of IQ, working memory or emotional intelligence, outperformed all other models we considered (see Tables S3 and S4 for details). 
Table 1 presents the results of the regression models estimating the propensity to engage in and recommend risky behaviours. Almost all credible effects reported in this table had Bayes factors exceeding 100, compared to the null hypothesis that there was no effect. One exception is the interaction between quadratic age and social norms in our participants propensity to recommend risky behaviour, which had a Bayes factor of 89 . These large Bayes factors speak for strong effects and hint to that we indeed were able to detect those within our sample.

We also examined whether we could replicate the direction of the effects in Table 1 on 1,000 synthetic data sets generated from the full posterior of the fitted models (post-hoc power). This was mostly the case with a replication probability of 1 , except for regression terms involving linear age fitted on our subjects' propensity to recommend desirable behaviours $\left(p_{\text {rep_age }}=.35 ; p_{\text {rep_age:HowManyOthers }}=.59\right)$. Taken together, these two Bayesian approaches converge and allow some confidence that the methods we used were adequate for the inferences reported in this article.

We found that perceived social norms positively affected the propensity to recommend both desirable and undesirable risks. In line with our expectations, individuals were more likely to recommend risky behaviours if they believed that many others engaged in those behaviours. Moreover, quadratic age predicted recommendation of undesirable risky behaviours and, to an even greater extent, desirable risky behaviours. We also found a negative interaction between perceived social norms and quadratic age, which implies that late adolescents were more likely to recommend risks of which they thought that others' do not frequently engage in. For desirable risks, this negative interaction was on top of a credible negative linear age effect, suggesting that the influence of descriptive social norms on recommendation behaviour generally decreased across adolescence. Perceived social norms also predicted participants' propensity to engage in risky behaviours. Notably, against our expectations, neither age nor quadratic age was a good predictor of engagement in desirable or undesirable risks. Also, we did not find a credible interaction between perceived social norms and quadratic or linear age regressors; in other words, there was no indication that adolescents are particularly sensitive to social norms (Figure 2).

Finally, the absence of evidence for a direct interaction of age with social norms on risk engagement notwithstanding, exploratory analysis suggests an adolescent-specific perception of social information; they report that others engage in risky behaviours $\left(b_{\text {quadratic_Age }}=11.50, \mathrm{CI}=[3.00,20.09]\right)$ more often than children or adults report. To investigate whether this quadratic trend might be indicative of an over estimation, we calculated to what extent individual judgments about the propensity of others' risk engagement deviates from the mean of our samples' self-reported propensity (Figure 3). Adolescents estimate overall deviates positively which could imply such overestimation; however, confirmatory studies are needed.

\section{Differences between engagement and recommendation}

As expected, participants' propensity to engage in risky behaviours generally differed from their propensity to recommend those behaviours (see Figure 1). We found opposite patterns of results for desirable and undesirable risks, which replicates a previous study using a similar approach (Helfinstein et al., 2015). That is, relative to their propensity to engage in risky behaviours, participants were less likely to recommend undesirable risks, but more likely to recommend desirable risks. This adds further evidence that desirable, 


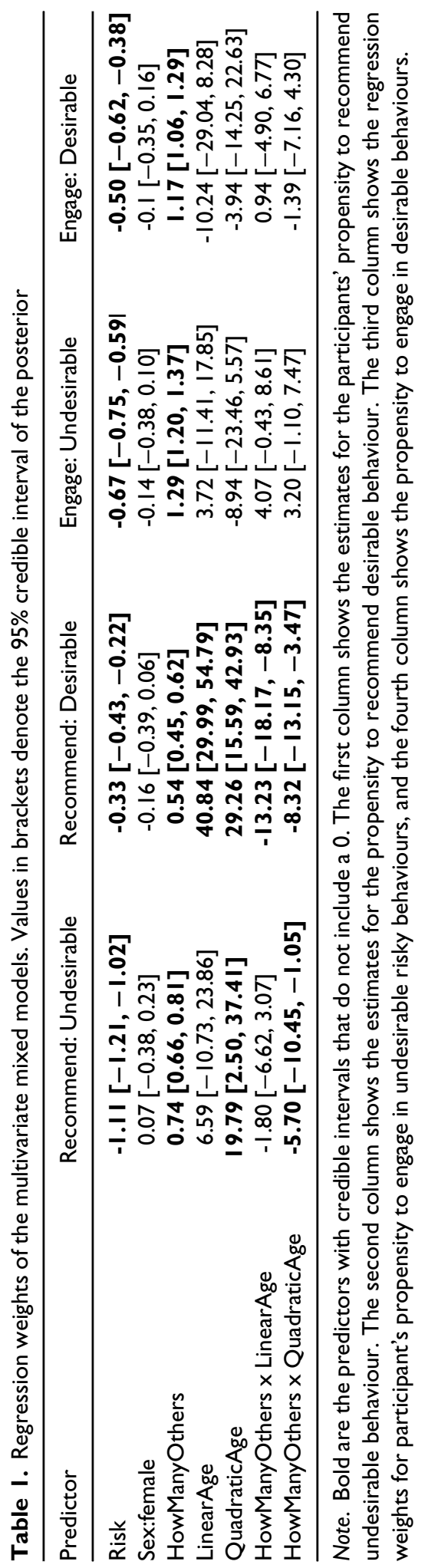


recreational risk-taking and maladaptive risk-taking may be distinct constructs (Duell \& Steinberg, 2019).

We also investigated how the differences between the decision to engage versus to recommend risky behaviours were related to our subjects' perception of risks and to their perception of social norms. Perceived risk contributed negatively to both engagement and recommendation. Notably, for undesirable risks, subjects' perception of risk reduced the propensity to recommend more strongly than the propensity to engage, as indicated by the non-overlapping credible intervals of the respective predictors. In order words, it appears as if people give more consideration to risks when recommending risky behaviours than when engaging in those behaviours themselves. Along similar lines, the
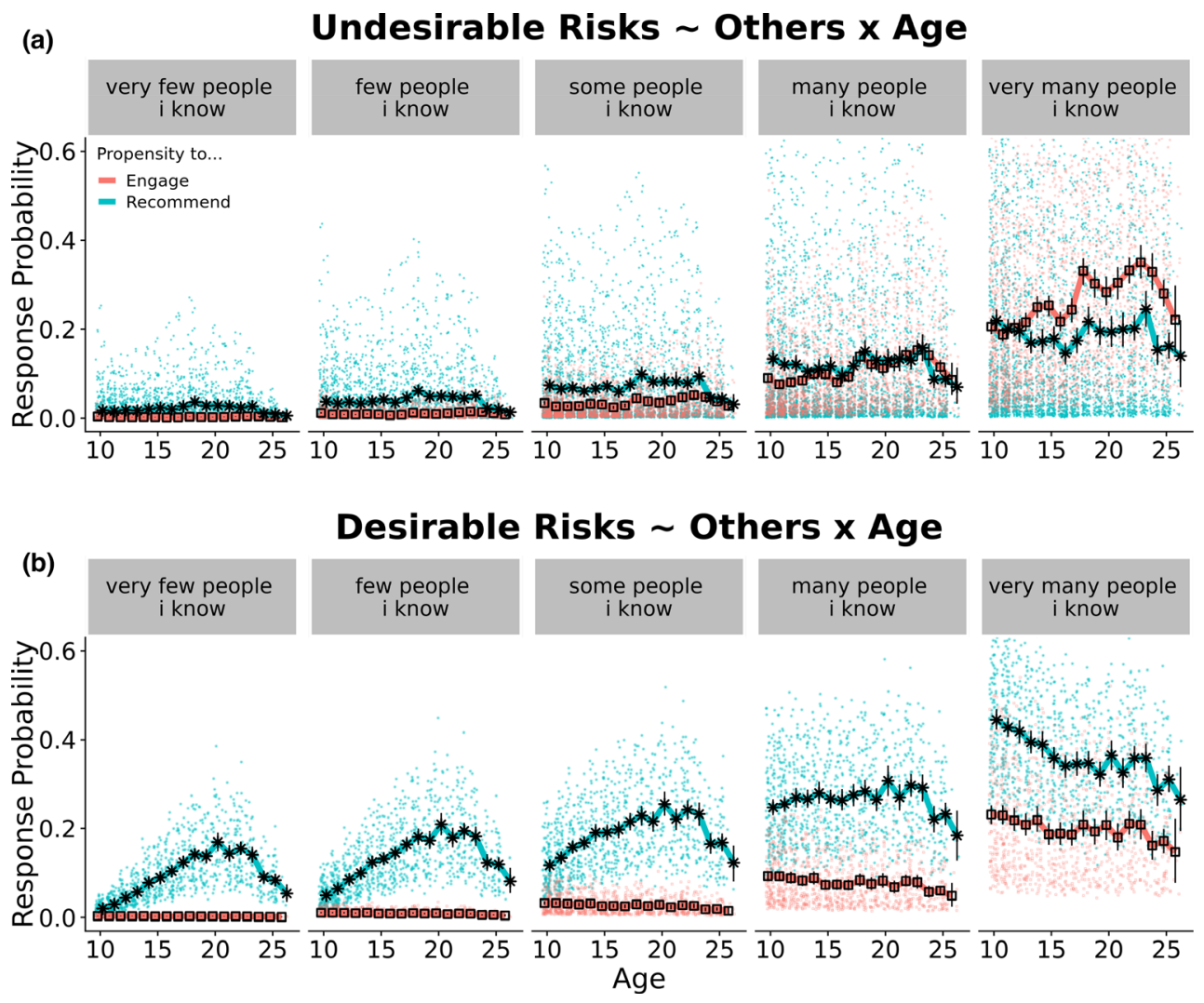

Figure 2. Posterior response probability for the strongest propensity to recommend (blue) and to engage in (red) desirable and undesirable risky behaviours by age and by strength of the descriptive norm. We show the posterior means, with the two-sided $95 \%$ confidence interval of the mean as error bars. Coloured dots refer to individual predictions under different covariate values. The panels visualize the interaction between perceived norms and age for risk engagement and risk recommendation. (a) Undesirable risks. As the perceived norm becomes stronger, adolescents are more likely to engage in risks than are other age groups (red line) but seem less likely to recommend them (blue line). (b) Desirable risks. There are no age differences in participants' propensity to engage in risky behaviours, but adolescents are more likely to recommend risky behaviours. As the perceived norm becomes stronger, this difference becomes smaller, as indicated by the negative quadratic interaction between perceived norms and age. [Colour figure can be viewed at wileyonlinelibrary.com] 
credible interval of the social norms predicting engagement in both desirable and undesirable risks was higher than and did not overlap with the credible interval of the social norms predicting recommendation of those behaviours. This implies, again in line with previous work, that subjects relied more strongly on social information when judging whether behaviours were appropriate for themselves than when judging whether they were appropriate for others.

\section{Discussion}

Adolescents are often seen as risk-takers, especially when in the company of their peers. Recent studies support the idea that directly observing and copying others can explain at least some of these social influence effects and that social influence may be stronger in younger individuals than in adults (Blankenstein et al., 2016; Braams et al., 2019; Ciranka \& van den Bos, 2019; Foulkes, Leung, Fuhrmann, Knoll, \& Blakemore, 2018; Gardner \& Steinberg, 2005; Knoll et al., 2015; Smith, Chein, \& Steinberg, 2014). However, these experiments addressed only part of the dynamics of social influence, leaving several questions unanswered. Here we addressed two of those questions based on crosssectional survey data. Specifically, we examined (1) whether adolescents reported an increased propensity to recommend risky behaviours and (2) whether adolescents' perception of social norms relates to their self-reported propensity to engage in risky behaviour. We found that adolescents were more likely to recommend risky behaviour than children or adults. However, in contrast to our expectations, we found no particular evidence of adolescent-specific sensitivity to social norms. Together, our results have implications for understanding peer influence in adolescent risk-taking and for attempts to attenuate the latter, some of which we discuss in the following.

\section{Do as I say? Risk recommendation}

Consistent with the findings of previous studies with adults (Helfinstein et al., 2015; Ubel, Angott, \& Zikmund-Fisher, 2011; Zikmund-Fisher, Sarr, Fagerlin, \& Ubel, 2006), our results showed that children and adolescents were also less likely to recommend than to engage in risky behaviours. Furthermore, they also focussed stronger on perceived risks when recommending behaviours than when engaging in them. Taken together, this suggests that the general pattern of more 'rational' thinking when recommending as compared to engaging in risky behaviours thus also holds for adolescents and children. However, adolescents were most likely to recommend risky behaviour. On top of that, our data suggest that adolescents were most likely to recommend risks that they thought were only infrequently taken by others (Figure 2). This trend that was stronger for desirable behaviours (e.g., parachuting or skiing), but also present in undesirable behaviours (e.g., riding without a helmet or getting drunk). That adolescents recommend risk-taking more strongly than people in other developmental stages could imply that they hold more favourable attitudes towards risk-taking. If adolescents believe that being a risk-taker is a valuable asset, they will also be more likely to endorse risky behaviour. Paired with adolescents' increased sensitivity to social signals (Blakemore \& Mills, 2014), their readiness to encourage others to take risks may well contribute to the rise of risky behaviours during this age period. These findings open up avenues for interventions that aim to attenuate the consequences of adolescent risk-taking. For instance, adolescents could be made more aware of their role and responsibility in spreading risky behaviours and asked to take the perspective of others more frequently. 


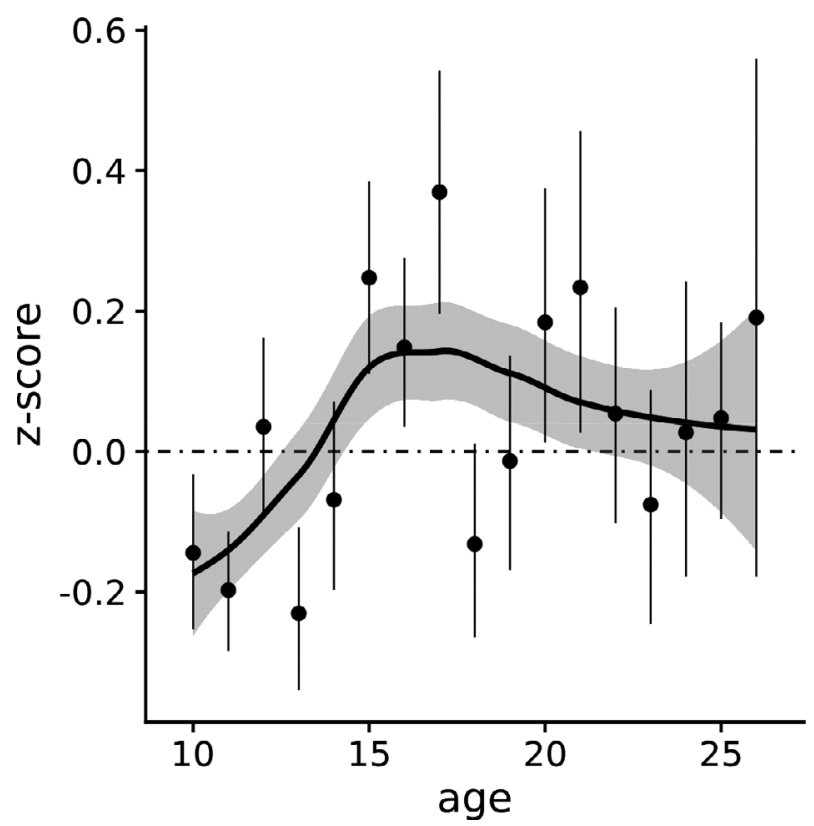

Figure 3. Over-and underestimation (positive and negative values, respectively) of others' engagement relative to the collected sample (y-axis) by age (x-axis). We show the mean and bootstrapped $95 \%$ confidence interval of the z-scored difference between the average self-reported propensity to engage in risky behaviours versus individual participants estimate of others' engagement by age. The fit line corresponds to locally estimated scatter plot smoothing with a bootstrapped $95 \%$ confidence interval.

\section{Do as others do? Risk engagement}

In line with previous findings, our data showed that - across age groups - perceived social norms were positively correlated with participants' self-reported propensity to engage in risky behaviour. That is, participants who believed that more of their peers engaged in a risky activity were more likely to report that they were willing to engage in that activity themselves. Contrary to our expectations, we found no interaction of age with perceived norms and propensity to engage in risky behaviours. Social norms were equally related to our participants willingness to engage in risky behaviour, regardless of age, implying that unobserved social norms may not have the outsized impact on adolescents as directly observed behaviour does. This suggests that the adolescent peak in social susceptibility is context dependent, (Crone \& Dahl, 2012), and I it is peer presence that affects adolescent decision-making processes the most (Albert \& Steinberg, 2011; Chein et al., 2011; Steinberg, 2005).

However, our data suggest that there is another way that social norms may have an outsized influence on adolescents. While other studies have shown that children and adults alike conform to social norms and adjust their conformity to an objective majority (Asch, 1955; Morgan \& Laland, 2012; Morgan, Laland, \& Harris, 2015), social norms often need to be inferred and cannot be directly observed (FeldmanHall \& Shenhav, 2019). Our post-hoc analysis suggests, that adolescents may be ill-calibrated when performing this inference. We show, that late adolescents estimated the general frequency of risky behaviours to be higher than other age groups and suggest that adolescents may overestimate others' engagement. As a result, the influence of social norms on risky 
behaviour may still be larger in adolescence; even though they are equally sensitive to norms, they have a greater tendency to overestimate the frequencies of risk behaviours. Taken together, these results have implications for interventions that aim at attenuating the consequences of adolescent risk-taking. Adolescents could be informed about true descriptive norms, a kind of intervention which has proved successful against bullying (Perkins, Craig, \& Perkins, 2011) and seems more effective than training resistance to peer pressure directly (Graham, Marks, \& Hansen, 1991).

Note that our result may also reflect a false consensus effect (Botvin, Botvin, Baker, Dusenbury, \& Goldberg, 1992). That is, the finding that those who engage in risky behaviours expect more others to engage in these behaviours as well (Brechwald \& Prinstein, 2011). Biased perception of consensus can itself contribute to change in future adolescent behaviour (Henry, Kobus, \& Schoeny, 2011). Thus, a false consensus effect can indirectly (via the perceived norm) contribute to the propensity to recommend and to engage in risky behaviours. Therefore, even if adolescents show a stronger false consensus effect, giving them information about actual peer norms will still be an effective intervention when it comes to attenuate the transmission of risky behaviour among adolescents.

\section{Limitations and future directions}

Because our study was not longitudinal, we cannot make causal claims about the relationship between social norms and risk-taking behaviour. While numerous experimental and longitudinal studies show a strong relationship between social norms and behaviour (Hansen \& Graham, 1991; Litt \& Stock, 2011; Perkins et al., 2011; Van Hoorn, Crone, \& Van Leijenhorst, 2017), further research is necessary to understand how social norms, risk-perception but also environmental factors impact risk-taking propensities across adolescence. For instance, some scenarios of our survey are illegal for minors, but not for adults. While minors de facto engage in activities that they would not be permitted to engage in (Moffitt, 2018), younger children still may perceive behaviours like drinking or stealing to be more risky than adults, simply because they are illegal for them and therefore recommend and engage in them less. As they grow older, breaking the law might appeal to thrill- and status-seeking adolescents. It is thus undoubtedly important to study which norms motivate young individuals to break which rules.

Further, our analysis is based on self-report data, which does not mimic the affective content of real-life risk-taking. As a result, we may have underestimated adolescent engagement in risky behaviour given that it is hypothesized that emotionally engaging situations are those where developmental differences are most likely to occur (Rosenbaum, Venkatraman, Steinberg, \& Chein, 2018).

Regardless of these caveats, it was recently demonstrated that self-reports capture reallife behaviour better than many experimental paradigms (Frey, Pedroni, Mata, Rieskamp, \& Hertwig, 2017; Hertwig, Wulff, \& Mata, 2019; Pedroni et al., 2017), demonstrating that they remain a valuable tool for understanding the development of risk-taking.

Further, while we focussed on risky behaviour, it is possible that adolescents are more likely to recommend any kind of behaviour or overestimate the prevalence of many other behaviours, too. Adolescent social susceptibility will be better understood if recommendation and conformity would be studied in non-risky domains as well.

The current study provides starting points for further investigating the relation between norms and behaviour in adolescence. For instance, when it comes to social 
impact, the source of social information is important, especially during adolescence (Knoll et al., 2015; Molleman et al., 2019). We asked our subjects to think about 'someone in general', when assessing their propensity to recommend risks, but did not give any specific instruction whom to think about. However, it is surely important whether subjects think about their friends or their parents (Knoll et al., 2015).

Future work should therefore ask individuals to think about specific groups of others or manipulate the presence, quality, and source of social norms in order to investigate which sources are used by individuals across development to construct their social norms. Finally, in this study we focused on descriptive norms - specifically, estimates of how often others engage in risky behaviours - and not on injunctive norms, which constitute judgements about the appropriateness of a behaviour (Cialdini \& Goldstein, 2004; McDonald \& Crandall, 2015). Future studies may benefit from eliciting perceptions of appropriateness, relating them to descriptive norms, and investigating the respective developmental trajectories across adolescence.

\section{Summary and Conclusion}

We identified two mechanisms that may contribute to the adolescent-specific increase in risky behaviours and the role of peers in it. We showed that although adolescents, like other age groups, are less likely to recommend risky behaviours than they are to engage in those behaviours themselves, they are more likely than children or adults to recommend risky behaviours. At the same time, while being similarly sensitive to social norms, adolescents might be overestimating the frequency of others' risk engagement. Although experimental and longitudinal studies are needed to better comprehend the determinants of these effects and their developmental course, it is likely that adolescents' increased propensity to recommend risky behaviours, paired with their overestimation of others' engagement in risky behaviours can trigger a vicious cycle that spreads risky behaviour through the adolescent population. However, our findings also show that adolescents do not mindlessly follow whatever they believe is normative behaviour - they may overestimate the normative character of some behaviours but likely use the same social heuristics as adults and children to decide whether to take risks. This offers the potential for interventions that aim at attenuating adolescent risk-taking behaviour: In line with successful attempts to reduce bullying or smoking in schools, our results suggest that informing adolescents about actual risk norms as compared to their perception of others' risk-taking can be utilized to advance health-promoting behaviour. Because after all, the very social mechanisms that contribute to greater risk engagement during adolescence can be used to foster and spread positive behaviours (Tankard \& Paluck, 2016).

\section{Acknowledgements}

We are grateful to Chantal Wysocki and Jann Wäscher for collecting the data despite all logistic difficulties. We are also grateful to Susannah Goss for editing the manuscript. SC is a predoctoral fellow of the International Max Planck Research School on Computational Methods in Psychiatry and Ageing Research (IMPRS COMP2PSYCH). The participating institutions are the Max Planck Institute for Human Development, Berlin, Germany, and University College London, London, UK. WB is supported by the Open Research Area (ID 176), the Jacobs Foundation, the European Research Council (ERC-2018-StG-803338), and the Netherlands Organization for Scientific Research (NWO-VIDI016.Vidi.185.068) 


\section{Conflicts of interest}

All authors declare no conflict of interest.

\section{Author contributions}

Simon Ciranka (Conceptualization; Data curation; Formal analysis; Investigation; Methodology; Software; Validation; Visualization; Writing - original draft) Wouter van den Bos (Conceptualization; Funding acquisition; Supervision; Writing - review \& editing).

\section{Data Availability Statement}

The data set generated and analysed during the current study, as well as the code to reproduce all analysis are available in a GitHub repository under, https://github.com/NomisCiri/Risk_Ga ps

\section{References}

Albert, D., \& Steinberg, L. (2011). Judgment and decision making in adolescence. Journal of Research on Adolescence, 21(1), 211-224. https://doi.org/10.1111/j.1532-7795.2010.00724.x

Asch, S. E. (1955). Opinions and social pressure. Scientific American, 193, 31-35. https://doi.org/ 10.1038/scientificamerican1155-31

Bandura, A., Ross, D., \& Ross, S. A. (1961). Transmission of aggression through imitation of aggressive models. The Journal of Abnormal and Social Psychology, 63, 575-582. https://doi. org/10.1037/h0045925

Blakemore, S.-J. (2008). The social brain in adolescence. Nature Reviews Neuroscience, 9, 267-277. https://doi.org/10.1038/nrn2353

Blakemore, S.-J., \& Mills, K. L. (2014). Is adolescence a sensitive period for sociocultural processing? Annual Review of Psychology, 65(1), 187-207. https://doi.org/10.1146/annurev-psych010213-115202

Blakemore, S. J., \& Robbins, T. W. (2012). Decision-making in the adolescent brain. Nature Neuroscience, 15, 1184-1191. https://doi.org/10.1038/nn.3177

Blankenstein, N. E., Crone, E. A., van den Bos, W., \& van Duijvenvoorde, A. C. K. (2016). Dealing with uncertainty: Testing risk- and ambiguity-attitude across adolescence. Developmental Neuropsychology, 41, 77-92. https://doi.org/10.1080/87565641.2016.1158265

Botvin, G. J., Botvin, E. M., Baker, E., Dusenbury, L., \& Goldberg, C. J. (1992). The false consensus effect: Predicting adolescents' tobacco use from normative expectations. Psychological Reports, 7O(1), 171-178. https://doi.org/10.2466/pr0.1992.70.1.171

Braams, B. R., Davidow, J. Y., \& Somerville, L. H. (2019). Developmental patterns of change in the influence of safe and risky peer choices on risky decision-making. Developmental Science, 22 (1), e12717. https://doi.org/10.1111/desc.12717

Brechwald, W. A., \& Prinstein, M. J. (2011). Beyond homophily: A decade of advances in understanding peer influence processes. Journal of Research on Adolescence, 21, 166-179. https://doi.org/10.1111/j.1532-7795.2010.00721.x

Brown, B. B., Clasen, D. R., \& Eicher, S. A. (1986). Perceptions of peer pressure, peer conformity dispositions, and self-reported behavior among adolescents. Developmental Psychology, 22(4), 521-530. https://doi.org/10.1037/0012-1649.22.4.521

Bürkner, P.-C. (2017). brms: An R Package for Bayesian Multilevel Models Using Stan. Journal of Statistical Software, 8O(1), 1-28. https://doi.org/10.18637/jss.v080.i01 
Carpenter, B., Gelman, A., Hoffman, M. D., Lee, D., Goodrich, B., Betancourt, M., ... Riddell, A. (2017). Stan: A probabilistic programming language. Journal of Statistical Software, 76(1), 1-32. https://doi.org/10.18637/jss.v076.i01

Carter, P. M., Bingham, C. R., Zakrajsek, J. S., Shope, J. T., \& Sayer, T. B. (2014). Social norms and risk perception: Predictors of distracted driving behavior among novice adolescent drivers. Journal of Adolescent Health, 54, S32-S41. https://doi.org/10.1016/J.JADOHEALTH.2014.01.008

Chein, J. M., Albert, D., O'Brien, L., Uckert, K., \& Steinberg, L. (2011). Peers increase adolescent risk taking by enhancing activity in the brain's reward circuitry. Developmental Science, 14, F1-F10. https://doi.org/10.1111/j.1467-7687.2010.01035.x

Cialdini, R. B., \& Goldstein, N. J. (2004). Social Influence: Compliance and conformity. Annual Review of Psychology, 55(1), 591-621. https://doi.org/10.1146/annurev.psych.55.090902. 142015

Ciranka, S., \& van den Bos, W. (2019). Social influence in adolescent decision-making: A formal framework. Frontiers in Psychology, 10, 1915. https://doi.org/10.3389/fpsyg.2019.01915

Crone, E. A., \& Dahl, R. E. (2012). Understanding adolescence as a period of social-affective engagement and goal flexibility. Nature Reviews Neuroscience, 13, 636-650. https://doi.org/ $10.1038 / \mathrm{nrn} 3313$

de Leeuw, J. R. (2015). jsPsych: A JavaScript library for creating behavioral experiments in a Web browser. Behavior Research Methods, 47(1), 1-12. https://doi.org/10.3758/s13428-014-0458-y

Duell, N., \& Steinberg, L. (2019). Positive risk taking in adolescence. Child Development Perspectives, 13(1), 48-52. https://doi.org/10.1111/cdep.12310

FeldmanHall, O., \& Shenhav, A. (2019). Resolving uncertainty in a social world. Nature Human Bebaviour, 3(5), 426-435. https://doi.org/10.1038/s41562-019-0590-x

Foulkes, L., Leung, J. T., Fuhrmann, D., Knoll, L. J., \& Blakemore, S.-J. (2018). Age differences in the prosocial influence effect. Developmental Science, 21(6), e12666. https://doi.org/10.1111/de sc. 12666

Frey, R., Pedroni, A., Mata, R., Rieskamp, J., \& Hertwig, R. (2017). Risk preference shares the psychometric structure of major psychological traits. Science Advances, 3(10), e1701381. https://doi.org/10.1126/sciadv.1701381

Gardner, M., \& Steinberg, L. (2005). Peer influence on risk taking, risk preference, and risky decision making in adolescence and adulthood: An experimental study. Developmental Psychology, 41, 625-635. https://doi.org/10.1037/0012-1649.41.4.625

Graham, J. W., Marks, G., \& Hansen, W. B. (1991). Social influence processes affecting adolescent substance use. Journal of Applied Psychology, 76(2), 291-298. https://doi.org/10.1037/00219010.76.2.291

Gullone, E., Moore, S., Moss, S., \& Boyd, C. (2000). The adolescent risk-taking questionnaire.Journal of Adolescent Research, 15(2), 231-250. https://doi.org/10.1177/0743558400152003

Hansen, W. B., \& Graham, J. W. (1991). Preventing alcohol, marijuana, and cigarette use among adolescents: Peer pressure resistance training versus establishing conservative norms. Preventive Medicine, 20(3), 414-430. https://doi.org/10.1016/0091-7435(91)90039-7

Helfinstein, S. M., Mumford, J. A., \& Poldrack, R. A. (2015). If all your friends jumped off a bridge: The effect of others' actions on engagement in and recommendation of risky behaviors. Journal of Experimental Psychology: General, 144(1), 12-17. https://doi.org/ 10.1037/xge0000043

Henry, D. B., Kobus, K., \& Schoeny, M. E. (2011). Accuracy and bias in adolescents' perceptions of friends' substance use. Psychology of Addictive Bebaviors, 25(1), 80-89. https://doi.org/10. $1037 / \mathrm{a} 0021874$

Hertwig, R., Wulff, D. U., \& Mata, R. (2019). Three gaps and what they may mean for risk preference. Philosophical Transactions of the Royal Society B: Biological Sciences, 374, 20180140. https:// doi.org/10.1098/rstb.2018.0140

Kandel, D. B. (1978). Homophily, selection, and socialization in adolescent friendships. American Journal of Sociology, 84, 427-436. https://doi.org/10.1086/226792 
Knoll, L. J., Magis-Weinberg, L., Speekenbrink, M., \& Blakemore, S.-J. (2015). Social influence on risk perception during adolescence. Psychological Science, 26, 583-592. https://doi.org/10.1177/ 0956797615569578

Kruschke, J. K. (2018). Rejecting or accepting parameter values in Bayesian estimation. Advances in Methods and Practices in Psychological Science, 1(2), 270-280. https://doi.org/10.1177/ 2515245918771304

Litt, D. M., \& Stock, M. L. (2011). Adolescent alcohol-related risk cognitions: The roles of social norms and social networking sites. Psychology of Addictive Behaviors, 25, 708-713. https:// doi.org/10.1037/a0024226

McDonald, R. I., \& Crandall, C. S. (2015). Social norms and social influence. Current Opinion in Behavioral Sciences, 3, 147-151. https://doi.org/10.1016/j.cobeha.2015.04.006

Megías-Robles, A., Gutiérrez-Cobo, M. J., Cabello, R., Gómez-Leal, R., Baron-Cohen, S., \& FernándezBerrocal, P. (2020). The 'Reading the mind in the Eyes' test and emotional intelligence'. Royal Society Open Science, 7, 201305. https://doi.org/10.1098/rsos.201305

Moffitt, T. E. (2018). Male antisocial behaviour in adolescence and beyond. Nature Human Behaviour, 2, 177-186. https://doi.org/10.1038/s41562-018-0309-4

Molleman, L., Kanngiesser, P., \& van den Bos, W. (2019). Social information use in adolescents: The impact of adults, peers and household composition. PLoS One, 14(11), e0225498. https://doi. org/10.1371/journal.pone.0225498

Morey, R. D., Hoekstra, R., Rouder, J. N., Lee, M. D., \& Wagenmakers, E.-J. (2016). The fallacy of placing confidence in confidence intervals. Psychonomic Bulletin \& Review, 23(1), 103-123. https://doi.org/10.3758/s13423-015-0947-8

Morgan, T. J. H., \& Laland, K. N. (2012). The biological bases of conformity. Frontiers in Neuroscience, 6, 87. https://doi.org/10.3389/fnins.2012.00087

Morgan, T. J. H., Laland, K. N., \& Harris, P. L. (2015). The development of adaptive conformity in young children: effects of uncertainty and consensus. Developmental Science, 18(4), 511-524. https://doi.org/10.1111/desc.12231

Nelson, E. E., Jarcho, J. M., \& Guyer, A. E. (2016). Social re-orientation and brain development: An expanded and updated view. Developmental Cognitive Neuroscience, 17, 118-127. https:// doi.org/10.1016/j.den.2015.12.008

Pedroni, A., Frey, R., Bruhin, A., Dutilh, G., Hertwig, R., \& Rieskamp, J. (2017). The risk elicitation puzzle. Nature Human Behaviour, 1, 803-809. https://doi.org/10.1038/s41562017-0219-x

Perkins, H. W., Craig, D. W., \& Perkins, J. M. (2011). Using social norms to reduce bullying. Group Processes E Intergroup Relations, 14, 703-722. https://doi.org/10.1177/1368430210398004

Petersen, A. C., Crockett, L., Richards, M., \& Boxer, A. (1988). A self-report measure of pubertal status: Reliability, validity, and initial norms. Journal of Youth and Adolescence, 17(2), 117-133. https://doi.org/10.1007/BF01537962

Pickett, C. L., Gardner, W. L., \& Knowles, M. (2004). Getting a cue: The need to belong and enhanced sensitivity to social cues. Personality and Social Psychology Bulletin, 30, 1095-1107. https:// doi.org/10.1177/0146167203262085

Ragan, D. T. (2016). Peer beliefs and smoking in adolescence: A longitudinal social network analysis. The American Journal of Drug and Alcohol Abuse, 42(2), 222-230. https://doi.org/10.3109/ 00952990.2015 .1119157

Reiter, A. M. F., Suzuki, S., O’Doherty, J. P., Li, S.-C., \& Eppinger, B. (2019). Risk contagion by peers affects learning and decision-making in adolescents, Journal of Experimental Psychology: General, 148, 1494-1504. https://doi.org/10.1037/xge0000512

Rosenbaum, G. M., Venkatraman, V., Steinberg, L., \& Chein, J. M. (2018). The influences of described and experienced information on adolescent risky decision making. Developmental Review, 47 , 23-43. https://doi.org/10.1016/j.dr.2017.09.003

Rouder, J. N., Morey, R. D., Verhagen, J., Province, J. M., \& Wagenmakers, E.-J. (2016). Is there a free lunch in inference? Topics in Cognitive Science, 8, 520-547. https://doi.org/10.1111/tops. 12214 
Schriber, R. A., \& Guyer, A. E. (2016). Adolescent neurobiological susceptibility to social context. Developmental Cognitive Neuroscience, 19, 1-18. https://doi.org/10.1016/j.dcn.2015.12.009

Shepherd, J. L., Lane, D. J., Tapscott, R. L., \& Gentile, D. A. (2011). Susceptible to social influence: Risky "driving" in response to peer pressure. Journal of Applied Social Psychology, 41, 773-797. https://doi.org/10.1111/j.1559-1816.2011.00735.x

Shulman, E. P., Smith, A. R., Silva, K., Icenogle, G., Duell, N., Chein, J., \& Steinberg, L. (2016). The dual systems model: Review, reappraisal, and reaffirmation. Developmental Cognitive Neuroscience, 17, 103-117. https://doi.org/10.1016/J.DCN.2015.12.010

Shulman, E. P., Steinberg, L. D., \& Piquero, A. R. (2013). The age-crime curve in adolescence and early adulthood is not due to age differences in economic status. Journal of Youth and Adolescence, 42, 848-860. https://doi.org/10.1007/s10964-013-9950-4

Smith, A. R., Chein, J., \& Steinberg, L. (2014). Peers increase adolescent risk taking even when the probabilities of negative outcomes are known. Developmental Psychology, 50, 1564-1568. https://doi.org/10.1037/a0035696

Somerville, L. H. (2013). The teenage brain. Current Directions in Psychological Science, 22(2), 121-127. https://doi.org/10.1177/0963721413476512

Somerville, L. H., Haddara, N., Sasse, S. F., Skwara, A. C., Moran, J. M., \& Figner, B. (2018). Dissecting "peer presence" and "decisions" to deepen understanding of peer influence on adolescent risky choice. Child Development, 90, 2086-2103. https://doi.org/10.1111/cdev.13081

Somerville, L. H., Jones, R. M., Ruberry, E. J., Dyke, J. P., Glover, G., \& Casey, B. J. (2013). The medial prefrontal cortex and the emergence of self-conscious emotion in adolescence. Psychological Science, 24, 1554-1562. https://doi.org/10.1177/0956797613475633

Steinberg, L. (2005). Cognitive and affective development in adolescence. Trends in Cognitive Sciences, 9(2), 69-74. https://doi.org/10.1016/J.TICS.2004.12.005

Steinberg, L. (2008). A social neuroscience perspective on adolescent risk-taking. Developmental Review, 28(1), 78-106. https://doi.org/10.1016/j.dr.2007.08.002

Steinberg, L., Icenogle, G., Shulman, E. P., Breiner, K., Chein, J., Bacchini, D., .. Takash, H. M. S. (2018). Around the world, adolescence is a time of heightened sensation seeking and immature self-regulation. Developmental Science, 21(2), e12532. https://doi.org/10.1111/desc.12532

Tankard, M. E., \& Paluck, E. L. (2016). Norm perception as a vehicle for social change. Social Issues and Policy Review, 10(1), 181-211. https://doi.org/10.1111/sipr.12022

Telzer, E. H. (2016). Dopaminergic reward sensitivity can promote adolescent health: A new perspective on the mechanism of ventral striatum activation. Developmental Cognitive Neuroscience, 17, 57-67. https://doi.org/10.1016/J.DCN.2015.10.010

Ubel, P. A., Angott, A. M., \& Zikmund-Fisher, B. J. (2011). Physicians recommend different treatments for patients than they would choose for themselves. Archives of Internal Medicine, 171(7), 630-634. https://doi.org/10.1001/archinternmed.2011.91

van de Bongardt, D., Reitz, E., Sandfort, T., \& Deković, M. (2015). A meta-analysis of the relations between three types of peer norms and adolescent sexual behavior. Personality and Social Psychology Review, 19(3), 203-234. https://doi.org/10.1177/1088868314544223

van den Bos, W., Crone, E. A., Meuwese, R., \& Güroğlu, B. (2018). Social network cohesion in school classes promotes prosocial behavior. PLoS One, 13(4), e0194656. https://doi.org/10.1371/ journal.pone.0194656

van Duijvenvoorde, A. C. K., Peters, S., Braams, B. R., \& Crone, E. A. (2016). What motivates adolescents? Neural responses to rewards and their influence on adolescents' risk taking, learning, and cognitive control. Neuroscience and Biobehavioral Reviews, 70, 135-147. https://doi.org/10.1016/j.neubiorev.2016.06.037

Van Hoorn, J., Crone, E. A., \& Van Leijenhorst, L. (2017). Hanging out with the right crowd: Peer influence on risk-taking behavior in adolescence. Journal of Research on Adolescence, 27, 189-200. https://doi.org/10.1111/jora.12265

Vehtari, A., Gelman, A., \& Gabry, J. (2017). Practical Bayesian model evaluation using leave-one-out cross-validation and WAIC. Statistics and Computing, 27, 1413-1432. https://doi.org/10.1007/ s11222-016-9696-4 
Wagenmakers, E.-J., Lodewyckx, T., Kuriyal, H., \& Grasman, R. (2010). Bayesian hypothesis testing for psychologists: A tutorial on the Savage-Dickey method. Cognitive Psychology, 60, 158-189. https://doi.org/10.1016/J.COGPSYCH.2009.12.001

Weiß, R. H. (1998). Grundintelligenztest Skala 2. CFT-20. Göttingen, Germany: Hogrefe.

Young, J. T. N., Rebellion, C. J., Barnes, J. C., \& Weerman, F. M. (2014). Unpacking the black box of peer similarity in deviance: Understanding the mechanisms linking Ppersonal behavior, peer behavior and perceptions. Criminology, 52(1), 60-86. https://doi.org/10.1111/1745-9125. 12029

Zikmund-Fisher, B. J., Sarr, B., Fagerlin, A., \& Ubel, P. A. (2006). A matter of perspective. Journal of General Internal Medicine, 21(6), 618-622. https://doi.org/10.1111/j.1525-1497.2006.00410.x

Received 21 July 2020; revised version received 20 January 2021

\section{Supporting Information}

The following supporting information may be found in the online edition of the article:

Figure S1. Posterior regression weights IQ, working memory and reading the mind in eye and their interactions with age for both, propensity to recommend (blue) and to engage (red).

Table S1. Willingness to engage. Shown are the item characteristics estimated on the base of our subjects' responses on the ARQ with all items.

Table S2. Willingness to recommend.

Table S3. Model comparison using leave one out cross validation for desirable risks.

Table S4. The same as table S3 but for undesirable risks. 